http://www.pakjas.com.pk

\title{
COMBINING ABILITY ANALYSIS OF PHYSIOLOGICAL TRAITS AND GRAIN YIELD IN MAIZE UNDER HEAT STRESS
}

\author{
Hafiz Muhammad Wasif Ali1 ${ }^{1, *}$, Muhammad Saleem ${ }^{1}$, Muhammad Ahsan' ${ }^{1}$ and Rashid Ahmad ${ }^{2}$ \\ ${ }^{1}$ Department of Plant Breeding and Genetics, University of Agriculture, Faisalabad-38040, Pakistan; \\ ${ }^{2}$ Department of Agronomy, University of Agriculture, Faisalabad-38040, Pakistan. \\ *Corresponding author's e-mail: wasifdogar@gmail.com
}

\begin{abstract}
Crop yield is reducing due to adverse effects of high temperature. Improvement of physiological status of maize plant against heat stress is important for a successful hybrid-breeding program. In the present study, six heat tolerant inbred lines, crossed in diallel mating design to develop $\mathrm{F}_{1}$ progeny. Parental lines including $30 \mathrm{~F}_{1}$ crosses were sown in triplicate randomized complete block design to select better performing inbred lines and $F_{1}$ crosses for leaf temperature, cell membrane thermostability, stomatal conductance, transpiration rate and grain yield under normal and heat stress environments. Low GCA/SCA variance ratio $(<1)$ showed the predominance of over-dominant gene action for all traits under normal and high temperature conditions, except osmotic potential (dominant) under high temperature. Three inbred lines, NCIL-20-4, D-135 and NCIL-10-5, showed desirable significant GCA estimates for physiological traits and grain yield under normal and high temperature. Five F $_{1}$ crosses, D-103 $\times$ NCIL-10-5 (its reciprocal), D-103 × NCIL-30-5, NCIL-20-4 × NCIL-10-5 and NCIL10-5 $\times$ NCIL-30-5, proved as the best single crosses for physiological traits and grain yield under heat stress. Selected inbred lines and $F_{1}$ crosses will be a good source for development of heat resilient, high yielding maize hybrids in future hybridbreeding programme.
\end{abstract}

Keywords: Cell membrane thermostability, diallel, grain yield, leaf temperature, stomatal conductance.

\section{INTRODUCTION}

Maize (Zea mays L.), a leading cereal worldwide and staple food of many countries, is a multipurpose crop used for human food, animal feed and industry purposes. Crop has wider genetic adaptability to grow under different agroecological environments. In Pakistan, sowing time of spring maize is during first fortnight of February. During these days, optimal climatic conditions favour healthy seed germination and seedling establishment. Anthesis usually begins during April. Maximum mean temperature reported during optimal environment was $36.1^{\circ} \mathrm{C} / 22.9^{\circ} \mathrm{C}$ (day/night). While under heat stress environment, maximum mean temperature was $42.3^{\circ} \mathrm{C} / 25.9^{\circ} \mathrm{C}$ (day/night) (Naveed et al., 2016). Chen et al. (2010) reported that when maize plant reaches up to $8^{\text {th }}$ leaf stage, it becomes susceptible to high temperature stress.

The average yield of maize in Pakistan is low because of various biotic and abiotic stresses. High temperature associated with drought causes decline in yield at reproductive and grain filling stages of the maize crop. Maize is cultivated over an area of 1334 thousand hectares with average yield being $4595 \mathrm{~kg} / \mathrm{ha}$. Area under cultivation increased by $12 \%$ comparable to little increase $(3.8 \%)$ in yield/ha. This increase in yield is mainly due to excessive use of fertilizers coupled with area under cultivation (Anonymous, 2016-17). Spring maize crop, faces heat stress at anthesis and grain filling (soft-dough/ milky stage) period which reduces pollen viability, silk receptivity and grain weight.

Plants are sensitive to high temperature at reproductive phase. High temperature beyond optimum level causes irreversible damages to plant growth, resulting in drop of crop productivity. Rapid changes in harsh climate resulted in huge decrease in cereal productivity, and so the world food production (Deryng et al., 2014). High temperature $\left(2^{\circ} \mathrm{C}\right)$ beyond threshold level has more drastic effects on crop yield compared to low (20\%) precipitation (Lobell and Burke, 2010; Shahid et al., 2017).

Plant's stomatal regulation depends on intensity and concentration of four major physiological factors (leaf temperature, light, hormones and leaf internal $\mathrm{CO}_{2}$ concentration). Among these leaf temperature is of prime importance. Under stress environment, leaf temperature, water relations \& stomatal conductance severely affect the plant growth. These three factors collectively known as "magic triangle" for plants (Valladares and Pearcy, 1997; Reynolds-Henne et al., 2010). High temperature coupled with limited water urges breeder to develop hybrids, which can withstand drought or heat stress without a remarkable decline in yield (Tester and Bacic, 2005). Few experiments based on research, to know the impact of high temperature on maize than other abiotic stresses. Previous research based on study of molecular and biochemical responses of maize plant at specific temperature under lab conditions using few 
genotypes, rather to study these effects under natural environment (Cairns et al., 2013).

The primary goal in developing heat tolerant hybrids is; to cope with heat stress at reproductive and grain filling stages and harvest a successful crop. Development of heat resilient cultivars is a challenging task. Narrow base genetic diversity of crop acts as major hindrance in achieving this goal. Breeders got few successes in development of heat tolerant genotypes, because genetic basis of heat tolerance about various agronomic and physiological traits is largely unknown (Driedonks et al., 2016). Heat tolerance is a complex and multigene inherited trait with slow progress because of $\mathrm{G} \times \mathrm{E}$ (Chen et al., 2012). The objective of this study was to select superior inbred lines and $F_{1}$ crosses on general combining ability and specific combining ability basis with higher grain yield under high temperature.

\section{MATERIALS AND METHODS}

The study was conducted at Department of Plant Breeding \& Genetics, University of Agriculture Faisalabad during the years 2014-2015. Faisalabad is about 189 meters high from sea level and lies in $31^{\circ}$ North (Latitude) and $73^{\circ}$ East (Longitude) region of the Punjab province, with semi-arid climate.

Six heat tolerant inbred lines, D-103, NCIL-20-4, D-135, NCIL-10-5, WA3748 and NCIL-30-5, were crossed using full diallel mating design in the field during autumn 2014. Both male and female inflorescences of maize plant were covered with kraft paper and butter paper bags, respectively to avoid foreign contamination. To ensure enormous seed setting, female parents were detasseled and pollinated manually. Parental lines and their $\mathrm{F}_{1}$ 's including reciprocal crosses were planted in the field during spring 2015 under normal and stress environment. The $1^{\text {st }}$ set was sown in the field during first fortnight of February under normal spring season while $2^{\text {nd }}$ set was sown during first fortnight of March, to expose high temperature stress at reproductive and grain filling stage. Experiments were conducted in randomized complete block design with three replicates. Each experimental unit comprised of two rows of $5.3 \mathrm{~m}$ each keeping row-to-row distances of $75 \mathrm{~cm}$ while plant-to-plant spacing was $23 \mathrm{~cm}$. To ensure good plant population, two seeds were dibbled per hill and thinning was done after 7 days from emergence to keep one healthy plant per hill. All agronomic and plant protection practices were kept uniform. Optimum irrigations applied to both normal and late sowing trials to avoid drought stress. Maximum mean temperature recorded for normal and heat stress experiments was $35.9^{\circ} \mathrm{C}$ and $40.4^{\circ} \mathrm{C}$, respectively. Ten randomly earmarked plants used for data recording of physiological and yield traits.

Physiological traits: Leaf temperature (LT) was recorded $\left({ }^{\circ} \mathrm{C}\right)$ from each experimental unit on a sunny day at 13.00$15.00 \mathrm{~h}$ by using infrared thermometer (RAYPRM $30 \mathrm{CFRJ}$,
RAYTEK, USA). For cell membrane thermostability (CMT), fully expanded leaves were used to collect $0.75 \mathrm{~cm}$ diameter round disks using sharp punch machine. The collected disks were washed thrice with de-ionized distilled water to remove surface adhered electrolytes. After washing, leaf discs were immersed in $10 \mathrm{~mL}$ of deionized water in glass tubes. One set of the tubes was put in water bath for one hour at $45^{\circ} \mathrm{C}$ and then both the sets were transferred in air- conditioned room at $22^{\circ} \mathrm{C}$ overnight. Next day, EC of each sample was measured by EC meter (LF 538) after shaking well the tubes. Samples were autoclaved for 15 minutes at $15 \mathrm{lbs}$ pressure and $121^{\circ} \mathrm{C}$ temperature to kill the leaf tissues and then allowed to cool down up to $22^{\circ} \mathrm{C}$ overnight. After autoclaving, EC was measured for the second time. Membrane integrity was measured by means of electrolyte leakage under both normal and stress environments. Percentage stability of leaf tissues was assessed from the first and second electrolyte measurements using the formulae (Sullivan, 1972).

Normal Temperature:

Cell membrane thermostability $=\left(1-\mathrm{C}_{1} / \mathrm{C}_{2}\right) \times 100$

High Temperature:

Cell membrane thermostability $=\left(1-\mathrm{T}_{1} / \mathrm{T}_{2}\right) \times 100$

Where, $\mathrm{C}_{1} \& \mathrm{C}_{2}$ are First and second conductivity measurement at room temperature $\left(22^{\circ} \mathrm{C}\right)$ before and after autoclave, respectively. $\mathrm{T}_{1} \& \mathrm{~T}_{2}$ are First and second conductivity measurement of plant sample treated at $45^{\circ} \mathrm{C}$ before and after autoclave, respectively.

The canopy temperature depression (CTD) was assessed by using portable hand held radiation thermometer (CHINO-IRAHOT) at anthesis stage on a bright sunny day between 12.00-15.00 h. Three readings were taken from each experimental unit by keeping instrument about $0.5 \mathrm{~m}$ above the canopy with roughly $30-60^{\circ}$ horizontally. Ambient air temperature was noted with hand held thermometer immediately after taking readings and CTD was calculated by following formula;

$$
\mathrm{CTD}=\mathrm{AT}-\mathrm{CT}
$$

where $\mathrm{AT}=$ ambient temperature, $\mathrm{CT}=$ canopy temperature Stomatal conductance $\left(g_{s}\right)$ and transpiration rate $\left(T_{r}\right)$ were measured from the second intact leaf from top of each plant using Hand-held Photosynthesis System (CI-340, CID, Inc. USA). Measurements were taken at noon during 11:00 -14:00 $\mathrm{h}$, with $\mathrm{PAR} \geq 1150 \mu \mathrm{mol} \mathrm{m} \mathrm{m}^{-2} \mathrm{~s}^{-1}$ on sunny days.

To measure leaf osmotic potential (OP), third leaf of plant were cut and frozen bellow $-20^{\circ} \mathrm{C}$ in a freezer for 7 days. After thawing, the cell sap was extracted with the help of a disposable syringe. The extracted sap was used to measure osmotic potential with the help of osmometer (Osmomat 030D, Cryoscopic osmometer printer, Genatec).

Osmotic potential (n) $=$ miliosmoles $\times 0.0832 \times$ Temp. $\left({ }^{\circ} \mathrm{K} / 10\right) \mathrm{MPa}$

where ${ }^{\circ} \mathrm{K}=$ Absolute temperature in Kelvin

Agronomic trait: For grain yield per plant (GYP), total weight of shelled grains was obtained from all the ears of each 
plot divided by the number of plants in that plot after drying to a constant moisture level (15\%).

Statistical analysis: Genetic variability among genotypes was estimated by subjecting data about various physiological and yield traits to analysis of variance (ANOVA) (Steel et al., 1997) using Minitab ${ }^{\circledR}$ version 17 (Minitab, 2014). Combining ability effects were estimated following Griffing's approach Method I Model I (Griffing, 1956).

\section{RESULTS}

The results of ANOVA showed significant genetic variability $(\mathrm{P} \leq 0.01)$ among the parents and crosses for all the traits under normal and heat stress conditions (Table 1).

Combining ability analysis: Combining ability ANOVA showed highly significant GCA \& SCA mean squares for all traits, and revealed the importance of both additive and nonadditive gene actions for trait inheritance under both experimental conditions (Table 2). Reciprocal effects for all traits were non-significant except for CMT. Cell membrane thermostability showed minor cytoplasmic effects under normal temperature.

General combining ability: Each inbred showed significantly GCA effects among traits (Table 3). Inbred D103 showed negative GCA estimates for LT, CTD, $\mathrm{g}_{\mathrm{s}}$, TR and GYP, while positive for CMT and OP under both temperatures. Two inbred lines, NCIL-20-4 \& NCIL-10-5 presented positive GCA effects for all traits except leaf temperature under normal and stress conditions. Inbred D-135 presented positive GCAs for CTD, $\mathrm{g}_{\mathrm{s}}, \mathrm{T}_{\mathrm{r}}$ and GYP, while negative GCA effects for LT \& CMT. Two inbred lines, WA3748 \& NCIL-30-5 showed negative GCA estimates for

Table 1. Mean squares of maize genotypes for various traits under normal and high temperature.

\begin{tabular}{|c|c|c|c|c|}
\hline \multirow[t]{2}{*}{ Traits } & \multicolumn{2}{|c|}{ NT } & \multicolumn{2}{|c|}{ HT } \\
\hline & GMS $(\mathrm{df}=35)$ & EMS $(d f=70)$ & GMS $(\mathrm{df}=35)$ & EMS $(d f=70)$ \\
\hline Leaf temperature & $3.524^{* *}$ & 0.0350 & $2.460^{* *}$ & 0.0710 \\
\hline Cell membrane thermostability & $43.075^{* *}$ & 0.9560 & $63.130^{* *}$ & 0.6470 \\
\hline Canopy temperature depression & $1.208^{* *}$ & 0.0220 & $1.183^{* *}$ & 0.0110 \\
\hline Stomatal conductance & $0.029^{* *}$ & 0.0010 & $0.013^{* *}$ & 0.0002 \\
\hline Transpiration rate & $0.803^{* *}$ & 0.0210 & $0.711^{\text {** }}$ & 0.0140 \\
\hline Leaf osmotic potential & $0.006^{* *}$ & 0.0001 & $0.010^{* *}$ & 0.0001 \\
\hline Grain yield per plant & $1367.130^{* *}$ & 14.7700 & $839.680^{* *}$ & 19.8200 \\
\hline
\end{tabular}

${ }^{* *}$ highly significant $\mathrm{p}=0.01, \mathrm{NT}=$ Normal temperature, $\mathrm{HT}=$ High temperature

Table 2. GCA, SCA and reciprocal mean squares of six maize inbred lines (diallel) under normal and high temperature.

\begin{tabular}{|c|c|c|c|c|c|c|}
\hline \multirow[t]{3}{*}{ Trait } & \multicolumn{6}{|c|}{ Mean Squares } \\
\hline & \multicolumn{2}{|c|}{ GCA (df = 5) } & \multicolumn{2}{|c|}{$\underline{\operatorname{SCA}(\mathrm{df}=15)}$} & \multicolumn{2}{|c|}{ Reciprocal (df=15) } \\
\hline & NT & HT & $\mathbf{N T}$ & HT & NT & HT \\
\hline Leaf temperature & $4.720^{* *}$ & $3.764^{* *}$ & $1.120^{* *}$ & $0.562^{* *}$ & 0.048 & 0.0960 \\
\hline Cell membrane thermostability & $66.953^{* *}$ & $97.641^{* *}$ & $9.493^{* *}$ & $15.755^{* *}$ & $1.700^{*}$ & 0.7920 \\
\hline Canopy temperature depression & $1.897^{* *}$ & $1.935^{* *}$ & $0.276^{* *}$ & $0.259^{* *}$ & 0.032 & 0.0160 \\
\hline Stomatal conductance & $0.044^{* *}$ & $0.021^{* *}$ & $0.007^{* *}$ & $0.003^{* *}$ & 0.001 & 0.0003 \\
\hline Transpiration rate & $1.227^{* *}$ & $1.025^{* *}$ & $0.193^{* *}$ & $0.197^{* *}$ & 0.023 & 0.0150 \\
\hline Leaf osmotic potential & $0.007^{* *}$ & $0.017^{* *}$ & $0.001^{* *}$ & $0.002^{* *}$ & 0.0003 & 0.0003 \\
\hline Grain yield per plant & $1732.33^{* *}$ & $1255.25^{* *}$ & $471.12^{* *}$ & $199.58^{* *}$ & 14.81 & 35.0800 \\
\hline
\end{tabular}

${ }^{* *}$ highly significant $\mathrm{p}=0.01,{ }^{*}$ significant $\mathrm{p}=0.05, \mathrm{NT}=$ normal temperature, $\mathrm{HT}=$ high temperature

Table 3. GCA effects of six parents under normal and high temperature.

\begin{tabular}{|c|c|c|c|c|c|c|c|c|c|c|c|c|c|c|}
\hline \multirow{2}{*}{$\begin{array}{l}\text { Inbred } \\
\text { Lines }\end{array}$} & \multicolumn{2}{|c|}{ LT } & \multicolumn{2}{|c|}{ CMT } & \multicolumn{2}{|c|}{ CTD } & \multicolumn{2}{|c|}{$\mathrm{g}_{\mathrm{s}}$} & \multicolumn{2}{|c|}{$\mathbf{T}_{\mathbf{r}}$} & \multicolumn{2}{|c|}{ OP } & \multicolumn{2}{|c|}{ GY } \\
\hline & NT & HT & NT & HT & NT & HT & NT & HT & NT & HT & NT & HT & NT & HT \\
\hline D-103 & -0.07 & $-0.20^{* * *}$ & 0.01 & $0.90^{* *}$ & $-0.22^{* *}$ & $-0.25^{* *}$ & $-0.02^{* *}$ & $-0.01^{*}$ & $-0.10^{* *}$ & -0.04 & $0.01^{* *}$ & $0.03^{* *}$ & -1.19 & $-3.50^{* * *}$ \\
\hline NCIL-20-4 & $-0.32^{* *}$ & $-0.18^{* *}$ & $3.22^{* *}$ & $2.77^{* *}$ & $0.24^{* *}$ & $0.29^{* *}$ & $0.04^{* *}$ & $0.04^{\text {** }}$ & $0.31^{\text {** }}$ & $0.24^{\text {** }}$ & $0.01^{* *}$ & $0.02^{\text {** }}$ & $4.58^{* *}$ & 0.14 \\
\hline D-135 & $-0.19^{* *}$ & $-0.17^{* *}$ & $-1.72^{* *}$ & $-0.52^{* *}$ & 0.04 & 0.033 & 0.001 & 0.001 & $0.07^{*}$ & 0.04 & $0.01^{* *}$ & $0.02^{* *}$ & $6.11^{* *}$ & $2.02^{*}$ \\
\hline NCIL-10-5 & $-0.82^{* *}$ & $-0.71^{* *}$ & $2.50^{* *}$ & $3.15^{\text {** }}$ & $0.66^{* *}$ & $0.64^{* *}$ & $0.09^{* *}$ & $0.06^{* *}$ & $0.38^{* *}$ & $0.39^{* *}$ & $0.03^{* *}$ & $0.04^{* *}$ & $15.38^{* *}$ & $18.42^{* *}$ \\
\hline WA 3748 & $0.98^{* *}$ & $0.92^{* *}$ & $-2.42^{* *}$ & $-4.20^{* *}$ & $-0.39^{* *}$ & $-0.42^{* *}$ & $-0.07^{* *}$ & $-0.05^{* *}$ & $-0.46^{* *}$ & $-0.40^{* *}$ & $-0.04^{* *}$ & $-0.05^{\text {** }}$ & $-20.05^{* *}$ & $-12.03^{* *}$ \\
\hline NCIL-30-5 & $0.42^{* *}$ & $0.35^{\text {** }}$ & $-1.58^{* *}$ & $-2.10^{* *}$ & $-0.32^{* *}$ & $-0.29^{* *}$ & $-0.05^{* *}$ & $-0.04^{* *}$ & $-0.20^{* * *}$ & $-0.24^{* *}$ & $-0.02^{* *}$ & $-0.02^{* *}$ & $-4.83^{* *}$ & $-4.77^{* *}$ \\
\hline $\operatorname{SE}\left(g_{i}\right)$ & 0.029 & 0.040 & 0.149 & 0.122 & 0.023 & 0.016 & 0.004 & 0.003 & 0.022 & 0.018 & 0.002 & 0.003 & 0.585 & 0.677 \\
\hline
\end{tabular}

$\mathrm{LT}=$ leaf temperature, $\mathrm{CMT}=$ cell membrane thermostability, CTD $=$ Canopy temperature depression, $\mathrm{g}_{\mathrm{s}}=\mathrm{stomatal}$ conductance, $\mathrm{T}_{\mathrm{r}}=$ transpiration rate, $\mathrm{OP}=$ Leaf osmotic potential, $\mathrm{GYP}=$ grain yield per plant, $\mathrm{SE}=\mathrm{Standard}$ Error 
all traits except LT under both temperatures. Based on GCA performance, four inbred lines D-103, NCIL-20-4, D-135 and NCIL-10-5 hade preferable GCA estimates almost for all traits, and favourable for using in future hybrid program.

Specific combining ability: Data pertaining to SCA of $30 \mathrm{~F}_{1}$ 's including reciprocal crosses, displayed in Table 4 \& 5, respectively. Three $F_{1}$ crosses, NCIL-20-4 $\times$ NCIL-10-5 along its reciprocal and NCIL-10-5 $\times$ D-103 showed negatively significant (desirable) SCA effects for LT under both temperatures. Three crosses, D-135 $\times$ NCIL-10-5, NCIL-20-4 $\times$ NCIL-10-5 and D-103 $\times$ NCIL-30-5 proved best specific combiner for CMT under both temperatures. Five crosses, D-103 $\times$ NCIL-10-5, NCIL-20-4 × NCIL-10-5, NCIL-20-4 $\times$ NCIL-30-5, D-135 $\times$ NCIL-30-5 and NCIL-10$5 \times$ WA3748, were best specific combiner for CTD under both temperatures.

Five crosses, D-103 $\times$ NCIL-20-4, D-103 $\times$ NCIL-10-5, D$103 \times$ NCIL-30-5, NCIL-20-4 $\times$ WA3748 and NCIL-10-5 $\times$
NCIL-30-5, were best specific combiners for $\mathrm{g}_{\mathrm{s}}$ under both temperatures. Five crosses, D-103 $\times$ NCIL-10-5, D-103 $\times$ WA3748, D-103 $\times$ NCIL-30-5, NCIL-20-4 $\times$ NCIL-30-5 and $\mathrm{D}-135 \times$ NCIL-10-5, were best for $\mathrm{T}_{\mathrm{r}}$ under both temperatures. Four $F_{1}$ crosses, D-103 $\times$ NCIL-10-5, NCIL20-4 $\times$ NCIL-10-5, D-135 $\times$ D-103 and WA3748 $\times$ NCIL-204 were best specific combiners with positively significant SCA estimates for OP under high temperature environment. Five crosses, D- $103 \times$ NCIL-10-5, D- $103 \times$ NCIL-30-5, NCIL-20-4 $\times$ NCIL-10-5, D-135 $\times$ WA3748 and NCIL-10-5 $\times$ NCIL-30-5, were best specific combiners for grain yield under both temperatures.

Gene action: GCA, SCA and reciprocal variances were estimated to know the predominance of additive or nonadditive gene action for trait inheritance (Table 6), under normal and heat stress condition. The SCA variances were

Table 4. SCA effects of $15 \mathrm{~F}_{1}$ 's (direct crosses) under normal and high temperature.

\begin{tabular}{|c|c|c|c|c|c|c|c|c|c|c|c|c|c|c|}
\hline \multirow[b]{2}{*}{ Cross Combinations } & \multicolumn{2}{|c|}{ LT } & \multicolumn{2}{|c|}{ CMT } & \multicolumn{2}{|c|}{ CTD } & \multicolumn{2}{|c|}{$\mathbf{g}_{\mathrm{s}}$} & \multicolumn{2}{|c|}{$\mathbf{T}_{\mathrm{r}}$} & \multicolumn{2}{|c|}{ OP } & \multicolumn{2}{|c|}{ GY } \\
\hline & NT & HT & NT & HT & NT & HT & NT & HT & NT & HT & NT & HT & NT & HT \\
\hline D-103 × NCIL-20-4 & $0.48^{* *}$ & $0.74^{* *}$ & -0.50 & $1.04^{* *}$ & $0.28^{* *}$ & $0.24^{* *}$ & $0.04^{* *}$ & $0.05^{* *}$ & 0.02 & 0.02 & $-0.02^{*}$ & $-0.05^{* *}$ & $3.38^{*}$ & $-3.16^{*}$ \\
\hline D-103 × D-135 & -0.07 & -0.19 & -0.27 & $0.71^{*}$ & 0.09 & $0.11^{*}$ & $-0.05^{* *}$ & 0.01 & -0.02 & 0.07 & 0.01 & -0.01 & -1.95 & 1.01 \\
\hline D-103 × NCIL-10-5 & $0.51^{* *}$ & -0.09 & 0.04 & 0.33 & $0.39^{* *}$ & $0.32^{* *}$ & $0.05^{* *}$ & $0.04^{* *}$ & 0.01 & $0.30^{* * *}$ & 0.01 & $0.02^{* *}$ & $8.91^{* *}$ & $6.52^{* *}$ \\
\hline D-103 × WA3748 & $0.52^{* *}$ & -0.35 & $0.99^{*}$ & $1.62^{* *}$ & $-0.28^{* *}$ & $-0.25^{* *}$ & $0.03^{*}$ & -0.01 & $0.41^{* *}$ & $0.19^{* *}$ & $-0.02^{*}$ & -0.01 & 1.23 & -2.67 \\
\hline D-103 $\times$ NCIL-30-5 & -0.07 & -0.10 & $1.96^{* *}$ & $1.90^{* *}$ & $-0.20^{* *}$ & $-0.20^{* *}$ & $0.05^{* *}$ & $0.03^{* *}$ & $0.26^{* *}$ & $0.19^{* * *}$ & 0.01 & 0.01 & $13.85^{* *}$ & $8.78^{* *}$ \\
\hline NCIL-20-4 × D & $-0.29^{* *}$ & 0.02 & 0.30 & $0.78^{*}$ & $-0.20^{* *}$ & $-0.12^{* *}$ & -0.01 & -0.0 & $0.17^{* *}$ & $0.17^{* * *}$ & $0.02^{* *}$ & 0.0 & $5.30^{* *}$ & -1.97 \\
\hline$-4 \times 1$ & -0.03 & $-0.21^{*}$ & $2.29^{* *}$ & $2.13^{* *}$ & $0.33^{* *}$ & $0.33^{* *}$ & 0.01 & 0.0 & $0.32^{* *}$ & 0.08 & $0.02^{* *}$ & $0.02^{* *}$ & $15.89^{* *}$ & $10.90^{* *}$ \\
\hline NCIL-20-4 × WA3748 & $0.58^{* * *}$ & $0.49^{* *}$ & $1.17^{* *}$ & -0.31 & $-0.23^{* *}$ & $-0.22^{* *}$ & $0.05^{* *}$ & $0.03^{* *}$ & $-0.12^{*}$ & 0.06 & $-0.03^{* *}$ & $-0.03^{* *}$ & -3.28 & $5.28^{* *}$ \\
\hline NCIL-20-4 × NCIL-30-5 & -0.04 & -0.02 & $1.45^{* *}$ & 0.51 & $0.33^{* *}$ & $0.36^{* *}$ & 0.01 & 0.01 & $0.25^{* *}$ & $0.34^{* *}$ & $0.03^{* *}$ & 0.01 & $8.09^{* * *}$ & 2.84 \\
\hline D-135 × NCIL-10-5 & $0.66^{* *}$ & 0.12 & $2.46^{* *}$ & $2.72^{* *}$ & $0.12^{*}$ & $0.14^{* *}$ & $0.04^{* *}$ & 0.0 & $0.25^{* *}$ & $0.16^{* *}$ & -0.01 & $-0.05^{* *}$ & -2.40 & 2.81 \\
\hline D-135 $\times$ WA3748 & $0.37^{* *}$ & 0.12 & -0.61 & $1.52^{* *}$ & 0.11 & 0.02 & $0.04^{* *}$ & $0.02^{* *}$ & $0.14^{*}$ & $0.10^{*}$ & $-0.02^{*}$ & 0.01 & $17.18^{* *}$ & $5.98^{* *}$ \\
\hline D-135 × NCIL-30- & $1.13^{* *}$ & $0.46^{* *}$ & -0.25 & $0.74^{*}$ & $0.51^{* *}$ & $0.48^{* *}$ & $0.03^{*}$ & & $0.18^{* *}$ & $-0.19^{* *}$ & $-0.02^{* *}$ & $-0.04^{* *}$ & $6.12^{* *}$ & $4.84^{* *}$ \\
\hline $\mathrm{NCI}$ & $0.39^{* *}$ & $0.71^{* *}$ & $1.20^{* *}$ & 0.45 & $0.32^{* *}$ & $0.32^{* *}$ & 0.01 & $-0 .($ & -0.02 & 0.01 & $-0.03^{* *}$ & $-0.03^{* *}$ & 1.9 & $5.65^{* *}$ \\
\hline NCIL-10-5 $\times$ & -0.06 & -0.08 & 0.56 & $2.17^{* *}$ & $-0.36^{* *}$ & $-0.31^{* *}$ & $0.04^{* *}$ & 0.0 & 0.05 & $0.19^{* *}$ & 0.01 & 0.01 & $7.75^{* *}$ & $7.84^{* *}$ \\
\hline $8 \times \mathrm{NCI} 305$ & $-0.45^{*}$ & $0.51^{*}$ & $1.48^{* *}$ & 0.33 & $0.23^{* *}$ & $0.23^{* *}$ & $-0.04^{* *}$ & 0.0 & -0.03 & 0.0 & $0.02^{* *}$ & -0.0 & 2.43 & $-5.82^{* *}$ \\
\hline $\operatorname{SE}\left(s_{\mathrm{ij}}\right)$ & 0.065 & 0.092 & 0.339 & 0.279 & 0.052 & 0.037 & 0.008 & 0.006 & 0.050 & 0.042 & 0.006 & 0.006 & 1.334 & 1.545 \\
\hline
\end{tabular}

Abbreviations are same as in Table 3.

Table 5. SCA effects of $15 \mathrm{~F}_{1}$ 's (reciprocal crosses) under normal and high temperature.

\begin{tabular}{|c|c|c|c|c|c|c|c|c|c|c|c|c|c|c|}
\hline \multirow[b]{2}{*}{ Cross Combinations } & \multicolumn{2}{|c|}{ LT } & \multicolumn{2}{|c|}{ CMT } & \multicolumn{2}{|c|}{ CTD } & \multicolumn{2}{|c|}{$\mathrm{g}_{\mathrm{s}}$} & \multicolumn{2}{|c|}{$\mathbf{T}_{\mathbf{r}}$} & \multicolumn{2}{|c|}{ OP } & \multicolumn{2}{|c|}{ GYP } \\
\hline & NT & HT & NT & HT & NT & HT & NT & HT & NT & HT & NT & HT & NT & HT \\
\hline NCIL-20-4 × D-103 & -0.03 & 0.15 & 0.08 & $0.85^{*}$ & 0.05 & 0.03 & 0.01 & 0.01 & $0.13^{*}$ & $0.12^{*}$ & 0.01 & -0.01 & 1.97 & -3.29 \\
\hline D-135 $\times$ D-103 & -0.02 & -0.05 & $-1.11^{*}$ & $-0.75^{*}$ & -0.03 & -0.05 & $0.04^{* *}$ & 0.01 & 0.08 & $0.15^{* *}$ & 0.01 & $0.02^{* *}$ & -1.52 & -3.60 \\
\hline D-135 × NCIL-20-4 & 0.01 & 0.08 & $-1.55^{* *}$ & -0.63 & -0.13 & -0.13 & $-0.04^{* *}$ & 0.01 & -0.02 & -0.02 & -0.01 & -0.01 & 1.43 & -0.63 \\
\hline NCIL-10-5 × D-103 & $-0.20^{*}$ & $-0.28^{*}$ & 0.41 & 0.56 & 0.05 & 0.07 & 0.01 & 0.01 & 0.10 & $0.15^{* *}$ & $0.02^{* *}$ & 0.01 & 1.15 & $5.31^{* *}$ \\
\hline NCIL-10-5 × NCIL-20-4 & 0.01 & $-0.25^{*}$ & 0.33 & 0.05 & 0.05 & 0.05 & $0.02^{*}$ & 0.01 & 0.08 & 0.03 & 0.01 & 0.01 & $-4.53^{* *}$ & 3.65 \\
\hline NCIL-10-5 × D-135 & -0.05 & -0.12 & $1.16^{*}$ & 0.50 & 0.10 & 0.13 & -0.01 & 0.01 & 0.10 & -0.03 & $0.02^{* *}$ & 0.01 & 2.72 & -1.22 \\
\hline WA3748 $\times$ D -103 & 0.08 & $0.45^{* *}$ & $-0.82^{*}$ & $-0.80^{*}$ & -0.07 & -0.08 & -0.01 & 0.01 & -0.02 & -0.05 & $-0.02^{* *}$ & -0.01 & $-3.23^{*}$ & -3.93 \\
\hline WA3748 $\times$ NCIL-20-4 & 0.05 & 0.18 & $-1.03^{*}$ & $-0.78^{*}$ & -0.08 & -0.08 & $-0.02^{*}$ & $-0.02^{* *}$ & $-0.17^{*}$ & -0.05 & -0.01 & $0.02^{* *}$ & -0.64 & -1.66 \\
\hline WA3748 $\times$ D-135 & 0.03 & 0.05 & -0.78 & -0.04 & -0.08 & 0.03 & 0.01 & $0.02^{* *}$ & 0.08 & $0.12^{*}$ & $-0.02^{* *}$ & -0.01 & $-3.91^{* *}$ & $-4.65^{*}$ \\
\hline WA3748 $\times$ NCIL-10-5 & 0.03 & 0.20 & $-0.95^{*}$ & $-1.06^{* *}$ & -0.22 & -0.17 & $-0.02^{*}$ & 0.01 & 0.10 & -0.05 & -0.01 & 0.01 & $-5.34^{* *}$ & $-9.89^{* *}$ \\
\hline NCIL-30-5 × D-103 & 0.13 & 0.07 & 0.02 & 0.21 & -0.05 & -0.05 & -0.01 & $-0.02^{* *}$ & 0.03 & -0.02 & $-0.02^{* *}$ & $-0.02^{* *}$ & -0.74 & -1.34 \\
\hline NCIL-30-5 × NCIL-20-4 & $0.37^{* *}$ & $0.27^{*}$ & $-1.28^{* *}$ & -0.59 & -0.23 & -0.08 & $-0.03^{*}$ & $-0.02^{* * *}$ & $-0.13^{*}$ & 0.07 & $-0.02^{* *}$ & $-0.03^{* *}$ & -0.37 & 0.40 \\
\hline NCIL-30-5 × D-135 & -0.03 & $0.32^{*}$ & $-0.87^{*}$ & -0.42 & -0.08 & -0.05 & -0.01 & 0.01 & 0.12 & -0.05 & $-0.02^{* *}$ & -0.01 & -2.74 & $-5.33^{* *}$ \\
\hline NCIL-30-5 × NCIL-10-5 & $0.38^{* *}$ & $0.27^{*}$ & $-1.37^{* *}$ & -0.35 & -0.27 & -0.13 & $0.02^{*}$ & -0.01 & 0.10 & $0.13^{*}$ & $-0.03^{* *}$ & $-0.02^{* *}$ & -2.23 & $-5.22^{*}$ \\
\hline NCIL-30-5 × WA3748 & -0.07 & 0.05 & 0.11 & $0.74^{*}$ & -0.05 & 0.05 & 0.01 & 0.01 & $0.18^{* *}$ & 0.01 & 0.01 & 0.01 & -1.74 & 0.88 \\
\hline $\operatorname{SE}\left(r_{i j}\right)$ & 0.077 & 0.109 & 0.399 & 0.328 & 0.061 & 0.043 & 0.010 & 0.007 & 0.062 & 0.049 & 0.007 & 0.007 & 1.569 & 1.818 \\
\hline
\end{tabular}

Abbreviations are same as in Table 3. 
Table 6. GCA $\left(\sigma_{\text {gca }}^{2}\right)$, SCA $\left(\sigma^{2}\right.$ sca $)$ and reciprocal $\left(\sigma^{2}\right.$ rec. $)$ variances in $6 \times 6$ diallel under normal and high temperature.

\begin{tabular}{lcccccccc}
\hline \multirow{2}{*}{ Trait } & \multicolumn{2}{c}{$\boldsymbol{\sigma}_{\text {gca }}$} & \multicolumn{2}{c}{$\boldsymbol{\sigma}_{\text {sca }}$} & \multicolumn{2}{c}{$\boldsymbol{\sigma}^{2}$ rec. } & \multicolumn{2}{c}{$\boldsymbol{\sigma}_{\text {gca }} / \boldsymbol{\sigma}^{2}$ sca } \\
\cline { 2 - 9 } & $\mathbf{N T}$ & $\mathbf{H T}$ & $\mathbf{N T}$ & $\mathbf{H T}$ & $\mathbf{N T}$ & $\mathbf{H T}$ & $\mathbf{N T}$ & $\mathbf{H T}$ \\
\hline Leaf temperature & 0.30000 & 0.26200 & 0.62900 & 0.28500 & 0.00700 & 0.01200 & 0.48000 & 0.92000 \\
Cell membrane thermostability & 4.73400 & 6.81200 & 4.95700 & 8.77200 & 0.37200 & 0.07200 & 0.95000 & 0.77000 \\
Canopy temperature depression & 0.13400 & 0.13900 & 0.14700 & 0.14400 & 0.00500 & 0.00300 & 0.91000 & 0.96000 \\
Stomatal conductance & 0.00300 & 0.00100 & 0.00400 & 0.00200 & 0.00020 & 0.00007 & 0.75000 & 0.50000 \\
Transpiration rate & 0.08500 & 0.07000 & 0.10000 & 0.11000 & 0.00100 & 0.00030 & 0.85000 & 0.64000 \\
Leaf osmotic potential & 0.00050 & 0.00100 & 0.00060 & 0.00100 & 0.00007 & 0.00007 & 0.83000 & 1.00000 \\
Grain yield per plant & 105.10 & 87.97 & 264.68 & 104.26 & 0.01700 & 7.63000 & 0.40000 & 0.84000 \\
\hline
\end{tabular}

higher than GCA variances for all the traits under both temperature regimes except for osmotic potential. Because of high SCA variances, GCA/SCA variance ratio was less than unity for all traits and indicated the predominance of nonadditive (dominance \& over-dominance) type of gene action. GCA \& SCA variances for leaf osmotic potential were equal under stress environment. Equal GCA/SCA variance ratio $(=1)$ declared the preponderance of dominant gene action for osmotic potential.

\section{DISCUSSION}

Assessment of GCA and SCA effects is necessary to identify and select the better performing inbred lines and $F_{1}$ crosses for each trait improvement. Results from combining ability ANOVA revealed significant GCA, SCA mean squares while reciprocal mean squares were non-significant. These results were opposed by Akbar et al. (2009) and Wattoo et al. (2014), who reported significant mean squares for these components (GCA, SCA and reciprocals) under normal and stress environment. Negative GCA and SCA estimates are favourable for leaf temperature, while positive GCA and SCA estimates preferred for remaining traits under normal and high temperature. It is also helpful to check either the best performing cross is combination of parents with high $\times$ high or high $\times$ low GCA effects. Non-additive genetic effects played important role for LT inheritance, as value of SCA variance was higher than GCA variance. Low GCA/SCA variance ratio $(<1)$ confirmed the findings about inheritance pattern of leaf temperature in maize (Ali et al., 2014). Additive genetic effects were important for LT under normal and high temperature (Naveed et al., 2016).

Plants grow under high temperature were more prone to irreversible damages to membrane integrity and resulting in cellular membranes instability (Nguyen and Joshi, 1992; Yildiz and Terzi, 2007). Significant genotypic variability observed for CMT among the inbred lines and $\mathrm{F}_{1}$ crosses as perceived by significant GCA and SCA genotypic mean squares under normal and high temperature. Low GCA/SCA variance ratio $(<1)$ for CMT showed that non-additive gene action played a major role for trait inheritance over additive gene action under normal and high temperature (Akbar et al., 2009; Ali et al., 2014). Inheritance of CMT was influenced by additive gene action as value of GCA/SCA variance ratio was higher than unity (Wattoo et al., 2014; Naveed et al., 2016). Non-additive genetic effects influenced inheritance pattern for stomatal conductance in maize, as ratio of GCA/SCA variance was lower than one (Mohamad et al., 2007; Ali et $a l ., 2014)$. Both GCA and SCA variances were significant for leaf transpiration rate in maize. Low GCA/SCA variance ratio $(\leq 1)$ revealed that non-additive genetic effects played a vital role for inheritance of leaf transpiration rate from parents to hybrids (Mohamad et al., 2007; Akbar et al., 2008; Ali et al., 2014). Results were against the findings of Wattoo et al. (2014) and Naveed et al. (2016), who reported significant role of additive genetic effects for $\mathrm{T}_{\mathrm{r}}$.

Leaf osmotic potential, maintained by accumulation of different types of osmo-protectants, produced in plants under high temperature and considered an important adaptive strategy of plants to cope against stress (Sakamoto and Murata, 2000). Osmo-protectants are helpful in maintaining leaf osmotic potential and protect the membranes of cellular organelles under heat stress environment (Farooq et al., 2008). GCA variance was less than SCA variance at normal temperature, so GCA/SCA variance ratio was less than unity, which proved predominance of non-additive gene action for trait inheritance under normal temperature. However, under high temperature regime GCA/SCA variance ratio was equal to unity indicating that dominant gene action were of great importance. The results revealed significance of additive and non-additive genetic effects for leaf osmotic potential under normal and high temperature, respectively (Akbar et al., 2008). Non-additive genetic effects were predominant for inheritance of grain yield as confirmed by low GCA/SCA variance ratio, which was less than unity (Hossein Haddadi $e t$ al., 2015; Akbar et al., 2009; Ali et al., 2014).

Conclusion: The results revealed significant role of both additive and non-additive gene action for all traits. However, reciprocal mean squares were insignificant showing absence of maternal effects for trait inheritance. The results proved that inbred lines having negative GCAs for leaf temperature, showed desirable GCA effects for other physiological traits and grain yield under both temperatures. So three inbred lines, NCIL-20-4, D-135 and NCIL-10-5 and five $\mathrm{F}_{1}$ crosses, D-103 $\times$ NCIL-10-5 (its reciprocal), D-103 × NCIL-30-5, NCIL-20- 
$4 \times$ NCIL-10-5 and NCIL-10-5 $\times$ NCIL-30-5 were earmarked as favourable for future hybrid breeding program. Combining ability analysis (variance components) showed the predominance of over-dominant gene action for all traits under normal and high temperature conditions, except OP (dominant) under high temperature. Selected parental lines and $F_{1}$ crosses could be used in hybrid breeding programme for development of heat resilient hybrids.

Acknowledgements: Corresponding author is highly thankful to Higher Education Commission, Pakistan for supporting his $\mathrm{PhD}$ studies.

\section{REFERENCES}

Akbar, M., M. Saleem, M.Y. Ashraf, A. Husain, F.M. Azhar and R. Ahmad. 2009. Combining ability studies for physiological and grain yield traits in maize at two temperature regimes. Pak. J. Bot. 41:1817-1829.

Akbar, M., M. Saleem, F.M. Azhar, M.Y. Ashraf and R. Ahmad. 2008. Combining ability analysis in maize under normal and high temperature conditions. J. Agric. Res. 46:27-38.

Ali, Q., A. Ali, M.F. Awan, M. Tariq, S. Ali, T.R. Samiullah, S. Azam, S. Din, M. Ahmad, M.N. Sharif, S. Muhammad, N.H. Khan, M. Ahsan, I.A. Nasir and T. Hussain. 2014. Combining ability analysis for various physiological, grain yield and quality traits of Zea mays L. Life Sci. J. 11:540-551.

Anonymous. 2016-17. Pakistan Economic Survey 2016-17. Finance and Economic Affairs Division, Ministry of Finance, Govt. of Pakistan, Islamabad, Pakistan.

Cairns, J.E., J. Crossa, P.H. Zaidi, P. Grudloyma, C. Sanchez, J.L Araus, S. Thaitad, D. Makumbi, C. Magorokosho, M. Bänziger, A. Mankir, S. Hearne and G.N. Atlin. 2013. Identification of drought, heat, and combined drought and heat tolerant donors in maize. Crop Sci. 53:13351346.

Chen, J., W. Xu, J.J. Burke and Z. Xin. 2010. Role of phosphatidic acid in high temperature tolerance in maize. Crop Sci. 50:2506-2515.

Chen, J., W. Xu, J. Velten, Z. Xin and J. Stout. 2012. Characterization of maize inbred lines for drought and heat tolerance. J. Soil Water Conserv. 67:354-364.

Deryng, D., D. Conway, N. Ramankutty, J. Price and R. Warren. 2014. Global crop yield response to extreme heat stress under multiple climate change futures. Environ. Res. Lett. 9:034011.

Driedonks, N., I. Rieu and W.H. Vriezen. 2016. Breeding for plant heat tolerance at vegetative and reproductive stages. Plant Reprod. 29:67-79.

Farooq, M., S. Basra, A. Wahid, Z. Cheema, M. Cheema and A. Khaliq. 2008. Physiological role of exogenously applied glycine betaine to improve drought tolerance in fine grain aromatic rice (Oryza sativa L.). J. Agron. Crop Sci. 194:325-333.

Griffing, B. 1956. Concept of general and specific combining ability in relation to diallel crossing systems. Aust. J. Biol. Sci. 9:463-493.

Ham, J.M., J.L. Heilman and R.J. Lascano. 1991. Soil and canopy energy balances of a row crop at partial cover. J. Agron. 83:744-753.

Hossein Haddadi, M., M. Esmaeilov, R. Choukan and V. Rameeh. 2015. Gene action and combing ability of some agronomic traits in corn using diallel analysis. Plant Breed. Seed Sci. 69:35-46.

Lobell, B. and M.B. Burke. 2010. On the use of statistical models to predict crop yield responses to climate change. Agric. For. Meteorol. 150:1443-1452.

Mohamad, A.A., A.N.M. Amin and S.I. Towfiq. 2007. Estimation of hetrosis, general and specific combining ability using diallel cross in maize (Zea mays L.) Mesopotamia J. Agric. 35:1-15.

Naveed, M., M. Ahsan, H.M. Akram, M. Aslam and N. Ahmed. 2016. Genetic effects conferring heat tolerance in a cross of tolerant $\times$ susceptible maize (Zea mays $\mathrm{L}$.) genotypes. Front. Plant Sci. 7:1-12.

Nguyen, H.T. and P.C. Joshi. 1992. Molecular strategies for the genetic dissection of water and high temperature stress adaptation in cereal crops. Proceedings of an International Symposium on the Adaptation of Food Crops to Temperature and Water Stress, Taiwan. pp.1318.

Reynolds-Henne, C.E., A. Langenegger, J. Mani, N. Schenk, A. Zumsteg and U. Feller. 2010. Interactions between temperature, drought and stomatal opening in legumes. Environ. Exp. Bot. 68:37-43.

Sakamoto, A. and N. Murata. 2000. Genetic engineering of glycine betaine synthesis in plants: Current status and implications for enhancement of stress tolerance. J. Exp. Bot. 51:81-88.

Shahid, M., M.F. Saleem, S.A. Anjum, M. Shahid and I. Afzal. 2017. Biochemical markers assisted screening of Pakistani wheat (Triticum aestivum L.) cultivars for terminal heat stress tolerance. Pak. J. Agri. Sci. 54:817825.

Steel, R.G.D., J.H. Torrie and D.A. Dicky. 1997. Principles and Procedures of Statistics: A biometrical approach, $3^{\text {rd }}$ Ed. McGraw Hill Book Co., New York, USA.

Sullivan, C.Y. 1972. Mechanisms of heat and drought resistance in grain sorghum and methods of measurement. In: N.G.P. Rao and L.R. House (eds.), Sorghum in the Seventies. Oxford and IPH publishing Co., New Delhi, India.

Tester, M. and A. Bacic. 2005. Abiotic stress tolerance in grasses: From model plants to crop plants. Plant Physiol. 137:791-793. 
Valladares, F. and R.W. Pearcy. 1997. Interactions between water stress, sun-shade acclimation, heat tolerance and photo-inhibition in the sclerophyll Heteromeles arbutifolia. Plant Cell Environ. 20:25-36.

Wattoo, F.M., M. Saleem and M. Sajjad. 2014. Identification of potential $F_{1}$ hybrids in maize responsive to water deficit conditions. Am. J. Plant Sci. 5:1945-1955.
Yildiz, M. and H. Terzi. 2007. Determination of high temperature stress tolerance by cell volume and photosynthetic pigmentation tests. Erciyes Univ. Sci. Inst. J. 23:47-60. 